Grammaire et enseignement du français langue étrangère et seconde - Permanences et ruptures du XVIe au milieu du XXe siècle (II)

\title{
L'apprentissage « autonome » du français proposé par un homme de lettres turc : Fransiz Grameri (1942) de Peyami Safa
}

Hanife Güven

\section{(2) OpenEdition}

Journals

\section{Édition électronique}

URL : https://journals.openedition.org/dhfles/3600

DOI : $10.4000 /$ dhfles.3600

ISSN : 2221-4038

Éditeur

Société Internationale pour l'Histoire du Français Langue Étrangère ou Seconde

Édition imprimée

Date de publication : 1 juin 2014

Pagination : 109-123

ISSN : 0992-7654

Référence électronique

Hanife Güven, «L'apprentissage « autonome » du français proposé par un homme de lettres turc Fransız Grameri (1942) de Peyami Safa », Documents pour l'histoire du français langue étrangère ou seconde [En ligne], 52 | 2014, mis en ligne le 02 août 2016, consulté le 28 mai 2021. URL : http:// journals.openedition.org/dhfles/3600 ; DOI : https://doi.org/10.4000/dhfles.3600

Ce document a été généré automatiquement le 28 mai 2021

(C) SIHFLES 


\title{
L'apprentissage « autonome » du français proposé par un homme de lettres turc : Fransiz Grameri ${ }^{1}$ (1942) de Peyami Safa
}

\author{
Hanife Güven
}

\section{Contexte historique}

1 Cet article comporte deux parties. La première est un rapide survol des grandes lignes du contexte historique des décennies 1930 et 1940 en Turquie. Cela nous permettra de mettre en évidence la conjoncture dans laquelle évolue la conception de l'enseignement des langues étrangères en général et de celui du français plus particulièrement. Dans la seconde partie nous focaliserons notre attention sur Peyami Safa, homme de lettres, et sur son ouvrage de grammaire en le comparant avec le manuel de français préconisé par le ministère de l'Éducation nationale. Cela nous permettra de mieux comprendre les circonstances qui l'ont fait naître.

2 La jeune République fondée en 1923 s'engage dans une reconstruction éducative et culturelle en affichant une volonté politique de rupture totale avec un passé semiféodal. Ainsi la fin de la décennie 1920 est ponctuée par l'adoption de la transcription latine en 1928. Par conséquent, la première moitié de la décennie 1930 voit une grande effervescence pour l'uniformisation linguistique accompagnée par une alphabétisation des masses et l'instauration du turc comme langue nationale du pays en la purifiant des éléments étrangers, surtout arabes et persans (Güven 2008).

Dans le domaine des langues étrangères, la méthode directe introduite officiellement dans les programmes dès l'avènement du régime de la Monarchie parlementaire en 1908 n'a pas répondu aux attentes. Pour l'ancrage dans le système éducatif des trois langues occidentales, français, allemand, anglais, les programmes de langues étrangères dans l'enseignement public sont revisités continuellement. Un rapide coup 
d'œil sur les programmes de français dans le premier et le second cycle (respectivement le collège et le lycée) peut nous permettre de faire un état de la situation.

4 Le premier cycle vise l'acquisition du français de base. Au bout d'une durée d'étude de trois ans à raison de 5 heures par semaine, les objectifs généraux à atteindre se résument comme suit (TCMV $1930: 53-54)$ :

-Lecture d'un texte simple qui ne contient pas de termes techniques ni de mots inconnus.

-Maîtrise d'un lexique (écrit et prononcé) composé de noms, verbes, adjectifs.

7 -Maîtrise et utilisation des règles de grammaire les plus importantes, pour s'exprimer oralement et à l'écrit.

8 -Rédaction de petites histoires, de courtes lettres de félicitations et de commande, d'un télégramme.

9 Les moyens pour permettre d'atteindre ces objectifs sont: des exercices de conversation, la dictée, la version, le thème sur des textes simples (anecdotes, petites histoires, chroniques de journaux, catalogues, listes de prix, affiches, annonces, factures). Les thèmes choisis sont liés à l'actualité des apprenants: école, vie quotidienne, courses, voyages, etc.

La recommandation ministérielle offre par ailleurs une liste de manuels qui méritent d'être proposés : Méthode Berlitz, Méthode Alge, France par Camerlynek [sic], Méthode Dent et les autres ouvrages dans la liste des manuels scolaires (TCMV 1930 : 55).

Dans le programme du deuxième cycle (TCMV 1934 : 88-91), pour les deux premières années, l'horaire hebdomadaire est de cinq heures. En classe terminale, les filières de lettres ont une heure de plus et celle des sciences une heure de moins, donc six et quatre respectivement. Les six grands moments de la classe communs aux trois années sont structurés autour d'activités telles que: lecture, conversation, récitation, grammaire, rédaction et traduction. En première et deuxième années s'y ajoute la dictée. En plus de la grammaire se présentent les analyses grammaticale et logique en deuxième année. Les fins et les moyens sont déterminés comme suit :

-études et analyses de textes classiques, avec une connaissance de la vie et de l'œuvre de l'auteur, -petites conversations sur les extraits étudiés ou d'autres sujets apportés par l'enseignant, possibilité de petits sketches adaptés au niveau des élèves, -analyse logique en détail, étude de la langue sur des textes difficiles,

15 -rédaction sur des sujets variés,

16 -récitation de morceaux choisis,

17 -versions et thèmes.

\section{Une nouvelle conception de la langue}

18 À partir de 1939, une mobilisation éducative débute avec l'entrée en fonction du ministre Hasan-Âli Yücel. L'un des thèmes de prédilection est de centraliser l'éducation avec un manuel unique pour chaque matière scolaire. Dans cette perspective, l'enseignement des langues et la formation des enseignants de langue sont 
sérieusement remis en question et subissent une révision en profondeur. Dans le cadre de la restructuration de la formation des maîtres pour le secondaire, l'école normale supérieure de Gazi diversifie son offre concernant les langues étrangères : sont créées les filières de français en 1941-1942, d'anglais en 1944-1945 et d'allemand en 1947-1948 (Demircan 1988 : 103).

19 Malgré les révisions, les constats d'échec se poursuivent chaque fois qu'on désire introduire une nouveauté dans les programmes. Ainsi le ministre rapporte en 1938 le discours tenu à propos de l'enseignement des langues à l'occasion du changement de programme de l'année 1919. Son but est de faire l'état des lieux et de justifier le fait que les résultats obtenus ne sont toujours pas satisfaisants vingt ans après :

On dit dans le programme que c'est une réalité indéniable que nos enfants n'arrivent pas à apprendre les langues à l'école. Par contre, parmi nos enfants qui dans les quartiers grecs et arméniens jouent avec leurs petits copains grecs et arméniens, le nombre de ceux qui apprennent ces langues rapidement est assez élevé. De même il est habituel de voir les petits apprentis des magasins qui viennent travailler dans nos quartiers sans en connaitre un mot apprendre le turc en quelques mois. Par conséquent dans l'enseignement des langues, la méthode la plus sûre est d'imiter la nature, c'est-à-dire d'adopter la méthode sans intermédiaire.

À propos de la décision prise pour la « Metot direct », on dit qu'on suit la méthode «Berliç ", que les livres et les programmes sont organisés selon ses principes, mais en dix ans elle n'a pas donné de bons résultats. On se plaint du fait que les anciens élèves connaissaient peu de vocabulaire et de règles et qu'ils savaient seulement un peu lire et écrire, mais ceux qui sont formés avec la méthode nouvelle ne savent rien d'autre que quelques phrases mémorisées et des formules stéréotypées. On explique la cause de cet échec en disant que la méthode directe en soi n'est pas une mauvaise méthode mais que ses conditions d'application n'ont pas pu être effectivement respectées. Le milieu de la classe étant artificiel par rapport au milieu naturel, cela constitue un handicap pour la réussite. (Yücel, 1994 : 180-181) [notre traduction]

Le plan d'étude fixé pour les langues étrangères est le résultat direct de ce conseil et se concrétise dans les manuels de français qui régissent la didactique du français jusqu'au milieu des années 1970. Ainsi commencent à paraître progressivement les premiers manuels officiels de français pour les six niveaux du secondaire.L'enseignement des langues vivantes dans les établissements secondaires est axé sur deux cycles de trois années. Le plan d'étude est affiché en détail dans les préfaces.

Dans les collèges, l'objectif fixé est de « donner aux élèves la disposition du vocabulaire concret usuel, la connaissance des règles élémentaires de la grammaire, la pratique de la conversation courante. »(Güçer et al. 1941 : IV).Les cours de français au lycée se fixent comme objectif $d$ '" assurer l'acquisition du vocabulaireabstrait des sentiments et des idées, une connaissance plus approfondie des règles essentielles de la grammaire, la pratique de la lecture, de la traduction et de la composition, enfin de les initier à la littérature française. "(Ibid.)

22 La préface du $\mathrm{V}^{\mathrm{e}}$ volume fait état de l'utilisation fonctionnelle de la langue et précise l'objectif général comme étant :

l'acquisition effective d'un instrument dont l'usage puisse être continué après la sortie du lycée, soit pour des besoins pratiques, soit pour l'information scientifique, soit pour des études littéraires. (Ibid.) 
En somme, tout porte à croire que la méthode directe dont se réclament les auteurs amènera les élèves «à la maîtrise effective du français, non seulement par la compréhension, mais encore par la parole. » (Ibid. : 4)

\section{Peyami Safa}

\subsection{Homme de lettres}

Peyami Safa (1899-1961) est le fils du célèbre poète Ismail Safa. Celui-ci meurt alors que son fils n'a que 2 ans. L'enfant grandit avec ses oncles paternels qui sont également des hommes de lettres. Il doit abandonner ses études secondaires et se retrouve dans le monde du travail à 13 ans. On le voit tour à tour employé au ministère de la Poste et du Télégramme, enseignant, traducteur, journaliste, auteur de manuels, polémiste et essayiste. Il a vécu tous les aléas de l'effondrement de l'Empire ottoman et de la formation de la République Turque (1923). L'auteur est une figure qui, en Turquie, fait autorité dès ses vingt ans pendant toute la première moitié $\mathrm{du} \mathrm{XX}^{\mathrm{e}}$ siècle. C'est par l'intermédiaire d'un dictionnaire Petit Larousse, offert comme cadeau decirconcisionparAbdullah $\operatorname{Cevdet}^{2}$, médecin militaire, penseur, poète, traducteur, fervent défenseur de la modernisation occidentale, que Peyami peut avoir un premier contact avec le français dès son plus jeune âge. Par la suite il a l'occasion d'améliorer son français et de l'apprendre à la perfection pendant qu'il travaille comme enseignant à Rehber-i Ittihat Mektebi (Ayvazoğlu 1998: 68)33. Son expérience dans cette école constitue le thème central de son roman Biz insanlar (Nous les hommes) (1959). Entre 1918-1961, il travaille comme journaliste et rédacteur en chef dans plusieurs journaux et revues. Son intérêt pour la littérature francophone se manifeste dans ses traductions $s^{4}$. Romancier très prolifique, essayiste, auteur de biographies, il débute très tôt sa carrière. Il écrit quinze romans, sept nouvelles, sans compter les romans policiers dont le nombre atteint les quatre-vingts volumes, principalement sous le pseudonyme de Server Bedii. Dans ses romans, il met en scène des personnages en quête d'identité avec des conflits intérieurs profonds, des personnages qui supportent mal le changement de civilisation, désorientés entre le traditionnel et le moderne, entre le superficiel et le profond, entre le temporaire et le spirituel, personnages aux prises avec leur mal-être et leur destin, tiraillés entre une vie corrompue, superficielle, limitée et matérielle et celle patiente, mature, consciente de sa propre identité.

\subsection{Pédagogue et auteur de manuels}

Peyami Safa ne pouvait pas échapper à des problèmes linguistiques, puisqu'il n'avait que 29 ans en 1928 quand la République de Turquie a adopté une nouvelle transcription pour sa langue. Il soutient d'une manière très active la mobilisation éducative en écrivant dès 1929 des alphabets, une dizaine de manuels de lecture et de grammaire pour l'enseignement du turc dans les écoles primaires. Comme le rappelle Korkmaz (1996: 5), les grammaires de la langue turque, écrites au début de l'ère républicaine, sont « des grammaires scolaires qui fixent les traits généraux de notre langue. »

Ses romans qui, au départ, matérialisent intensément les problèmes identitaires, transmettent par ailleurs, petit à petit, une culture éducative nouvelle. Ainsi, dans l'un de ses romans intitulé Yalnızız (Nous sommes seuls) (1951), le protagoniste Samim écrit 
un roman où il imagine un pays utopique dans cent cinquante ans, du nom de Simeranya. L'éducation rêvée reflète quasi une nouvelle conception de l'enseignement qui va à l'encontre du paradigme dominant :

En Simeranya, il y a des salles de lecture, des laboratoires, des ateliers, des maisons de musique, de théâtre, de cinéma et de sport, pour tous les niveaux. Des hommes de tous âges les fréquentent. Ils étudient tout seuls chaque matière qui les passionne et ils les acquièrent. Pour les enfants et les jeunes il y a des enseignants guides qui leur montrent les méthodes de recherche. Leur mission n'est pas d'enseigner mais de leur apprendre les méthodes d'apprentissage. Car la pédagogie en vigueur en Simeranya reconnaît que ce que l'homme apprend pendant toute sa vie, il ne l'apprend qu'au moment où il veut l'apprendre et à la suite de ses recherches personnelles. Mais dans les écoles anciennes, c'est-à-dire du monde d'aujourd'hui par rapport à Simeranya, on ne trouve plus de trace d'écoles classiques puisqu'on reconnaît que tout ce qu'on enseigne aux enfants et aux jeunes ne sert à rien, tant que cela ne répond pas à des capacités et à des besoins déterminés: [en Simeranya] il n'y a pas de classe, de bureau, de programme, d'enseignant et de professeur d'université qui font des cours magistraux, comme s'ils faisaient des discours. Pas de diplômes. (Yalnızız 2008 : 30) [notre traduction]

\subsection{Fransız Grameri, 1942} départ des mots courants, des phrases élémentaires isolées, des questions-réponses sur le modèle de la méthode Berlitz. La démarche utilisée pour enseigner la grammaire est la grammaire explicite et déductive. La langue est présentée aux apprenants à travers des mots et des propositions fabriqués, très proches de la langue parlée par leur brièveté et simplicité. Seuls, à la toute dernière leçon, trois petits poèmes avec des vers très courts apparaissent. La progression est linéaire, fixée a priori, d'une manière rigoureuse et contraignante. Elle traite les points grammaticaux les uns après les autres en partant de ceux qui sont censés être les plus faciles et les plus simples. Chaque unité est organisée en deux temps : dans un premier temps l'objectif est de faire saisir à l'apprenant un point grammatical qui est annoncé dans le titre. Dans un deuxième temps ce point grammatical est décrit et vérifié. moments bien distincts que le sous-titre en encadré rapporte : lecture - vocabulaire - 
traduction - grammaire - exercices - conversation. La progression est basée sur neuf catégories grammaticales ${ }^{6}$. L'auteur précise dans la dernière leçon : «La proposition se divise en plusieurs types. Mais dans ce petit précis nous ne trouvons pas nécessaire de donner plus d'information théorique »(176). Quelle autonomie pourrait assurer un manuel de grammaire surtout s'il s'agit de l'apprentissage d'une langue assez lointaine de la langue officielle/maternelle? souligner très succinctement les principales caractéristiques du turc en retenant les zones les plus problématiques par rapport au français pour mettre en évidence les différences, pour ne pas dire les difficultés qui se présentent à un turcophone. D’abord le turc est une langue agglutinante qui fonctionne par une succession de suffixes 
s'ajoutant à la racine ou à un autre suffixe. Elle n'a pas d'article, ni de distinction de genre. Le pluriel n'est qu'une terminaison euphonique. Le système possessif et la déclinaison des cas sont prédominants dans le système grammatical. Les déterminants précèdent les noms. Le verbe être n'est qu'une copule qui n'a que quatre formes pour quatre temps, le reste étant exprimé par un autre verbe (devenir). Le verbe avoir se rend en français par le nom conjugué au possessif avec la formule il y a qui suit. La concordance des temps n'existe presque pas dans les subordonnées. Trois suffixes (pour le passé, le présent et le futur) qui tiennent la place de conjonctions de subordination suffisent à relier une proposition à une autre. Il a trente-deux temps dont l'oui-dire qui n'existe pas en français.

Il est trivial de nos jours d'affirmer que la langue première de l'apprenant joue un rôle dans l'acquisition de la LE. Quand le ministre Yücel compare la compétence en langue étrangère des enfants grecs et arméniens avec celle des enfants turcs et qu'il affirme qu'on n'arrive pas" à enseigner à l'école » les langues étrangères, il est clair qu'il ne prend pas en compte le bilinguisme précoce ni l'origine indo-européenne des langues maternelles des premiers, alors que pour les seconds l'écart entre le système de leur LM et celui de la L2 est grand.

Maintenant, examinons l'unique manuel de français proposé par le ministère de l'Éducation nationale. On constate de prime abord que l'avertissement fait état de trois grandes lois dans la "conquête d'une langue »: intuition, instinct d'imitation, répétition (Güçer et al. 1940: 1). Il y est conseillé d'éviter la traduction «source d'erreurs et d'habitudes défectueuses ». La progression suit un ordre grammatical basé, soit sur un thème de la vie quotidienne, soit sur une catégorie grammaticale. Bien que non distinctes et non régulières, les différentes phases qui reviennent pour les unités didactiques sont : prononciation et lecture - grammaire - dictée - récitation - exercices - devoirs - phrases nouvelles, etc. Plusieurs tableaux colorés servent à introduire le vocabulaire sans avoir recours à la langue maternelle. Pour certains cours sont indiqués les objets nécessaires à apporter en classe. Les auteurs suivent les principes de la méthodologie active qui préconisent d'enseigner une grammaire inductive et d'inculquer à l'apprenant les structures de base de la langue orale avant de passer à la graphie.

41 À la lumière de cette situation, pour peu qu'on compare les deux ouvrages, un parallélisme manifeste peut être constaté sur le plan du contenu et on est frappé par la conception d'une langue assez descriptive. La différence réside principalement dans l'usage de la langue maternelle dans Fransiz Grameri de Safa. Bien que sans avantpropos, ni introduction, la conception mise en œuvre n'est pas difficile à déceler. Il offre un savoir grammatical et un savoir lexical avec leur traduction en LM, pour faire prendre conscience du fonctionnement interne de la L2. Les grammaticalisations de la L1 et de la L2 étant assez différentes, les explications en langue maternelle viennent confirmer les hypothèses de l'apprenant et permettent de le sécuriser. En cela on peut supposer que son ouvrage est une compensation, grâce à l'usage de la langue maternelle, des insuffisances éventuelles du manuel officiel. Il est gratifiant d'avoir un instrument de formation complémentaire qui tienne compte de la différence des codes linguistiques. Puisque l'analyse grammaticale en langue maternelle commence dès la $3^{\mathrm{e}}$ primaire, l'apprenant est donc invité avec cet ouvrage à engager une réflexion métalinguistique sur le fonctionnement de la langue. La terminologie grammaticale, comme il est précisé en sous titre est «facile». L'élève participe activement à la 
construction de son savoir en associant ce qu'il sait sur sa langue et les universaux en général. L'unique contradiction réside dans le fait que cet instrument présuppose un enseignant dans les premières phases de l'apprentissage alors que la notion d'« autonomie » correspondrait plutôt à un apprentissage « tout seul », individuel, de la langue.

Après l'approche communicative, de nos jours l'approche interculturelle semble avoir redécouvert cette réalité que la L1 est un filtre par lequel passe tout apprenant. Safa, en "humble artisan", apporte avec ce livre sa contribution personnelle au processus d'apprentissage du français et ainsi à la modernisation occidentale de son pays. Sa démarche va dans le sens de la psychologie cognitive dont l'apport dans le processus d'apprentissage est la prise en compte des connaissances antérieures de l'élève (Tardif 1992). Le nouveau système linguistique se construira en s'articulant aux connaissances acquises (explicites ou implicites) antérieurement (Gaonac'h 1987). L'ouvrage présuppose donc que le sens naît grâce à la traduction et qu'il faut faire prendre conscience à l'apprenant des contraintes auxquelles il doit faire face: les règles de fonctionnement de la langue-cible.

\section{Conclusion}

C'est un fait bien connu que le sentiment général qui règne dans les milieux scolaires turcs est que le français est une langue difficile aussi bien en tant que moyen de communication que de savoir explicite. Il est vrai que le manuel officiel préconisé par les programmes scolaires découle des méthodologies actives pour lesquelles l'objectif principal est l'acquisition d'un savoir lexical et grammatical sans avoir recours à la LM. Fransiz grameri de Safa est une grammaire pédagogique qui se concentre également sur l'objet, mais propose une stratégie d'enseignement différente, à savoir : le recours à la langue maternelle.

44 Si l'autonomie signifie la capacité de prendre en charge son propre apprentissage (Holec 1979 : 4),l'autonomie« supposée »du manuel de grammaire de Safa est donc rendue possible par la pratique de la traduction, par l'explication des règles assez allégées de la langue-cible et par le recours à des activités de questions-réponses qui vont conduire l'apprenant à une prise de conscience concernant le fonctionnement de la L2. De la sorte, le processus d'apprentissage de la L2 est facilité et accéléré. Cette stratégie explicite pour asseoir les connaissances lexicales et morpho-syntaxiques de base sera d'autant plus gratifiante que le manuel officiel utilisé n'y a pas recours.

Tout en étant conscient que cette pratique ne mène pas à une conception de la langue comme moyen de communication et que le savoir explicite ne peut pas se transformer en habileté à communiquer, comme l'affirme désormais la neurolinguistique, il n'empêche pas moins que la LM permet aux apprenants de prendre conscience des différences entre les deux systèmes linguistiques et d'en tirer profit pour améliorer leur apprentissage de la L2 à l'école, apprentissage implicite, limité à la supposition et à l'intuition. À notre avis, l'ouvrage de Safa présente l'intérêt d'être rédigé par un observateur minutieux et offre des réponses qui assurent une meilleure connaissance d'une L2 dont les concepts sont assez éloignés de la LM de l'apprenant. L'autonomie "supposée » signifierait ici donc l'apprentissage individuel de la L2 complémentaire d'un cours traditionnel dans les milieux scolaires. 


\section{BIBLIOGRAPHIE}

AYVAZOGLU, Beşir (1998). Peyami, Hayatı Sanatı Felsefesi Dramı [Peyami, sa vie, son art, sa philosophie].Istanbul : Ötüken.

BESSE Henri (1985). Méthodes et pratiques des manuels de langue.Paris : Didier.

DEMIRCAN, Ömer (1988). Türkiye'de Yabancı Dil [Langue étrangère en Turquie]. Istanbul : Remzi Kitabevi.

GAONAC'H, Daniel (1987). Théorie d'apprentissage et acquisition d'une langue étrangère. Paris : LAL Hatier CREDIF.

GÜÇER, Behçet, BERGEAUD, COUDERT, ÜN, İzzet Hamit, ROBIN (1940). Fransızca Ders Kitabı I. [Manuel de français I].Istanbul : Maarif Basımevi.

GÜÇER, Behçet, BERGEAUD, Camille, ÜN, İzzet Hamit (1941). Fransızca Ders Kitabı V. [Manuel de français V].Istanbul : Maarif Basımevi.

GÜVEN, Hanife (2008). « L'instrumentalisation politique d'une langue : la latinisation de l'alphabet ». In Geneviève Zarate, Danielle Lévy, Claire Kramsch (dir.), Précis du plurilinguisme et du pluriculturalisme. Paris : Éd. des Archives contemporaines, 419-424.

HOLEC, Henri (1979). Autonomie et apprentissage des langues étrangères. Strasbourg : Conseil de la coopération culturelle du Conseil de l'Europe, Hatier.

KORKMAZ, Zeynep (1996). « Gramer Konularımızla İlgili Bazı Sorunlar ».[Quelques problèmes concernant nos sujets de grammaire]. Türk Dili (535), 3-18.

SAFA, Peyami (1942). Fransız Grameri : Okuma-lügat-tercüme-gramer-tatbikat ve konuşma.[Grammaire $\mathrm{du}$ français : lecture-lexique-traduction-grammaire-exercices et conversation].Istanbul : Cumhuriyet Kitabevi.

SAFA, Peyami (2008 [1959]). Biz insanlar.[Nous les hommes]. Istanbul : Alkım.

SAFA, Peyami (2008 [1951]). Yalnızız.[Nous sommes seuls]. Istanbul : Alkım.

TARDIF, Jacques (1992). Pour un enseignement stratégique. L'apport de la psychologie cognitive. Paris : Les Éditions Logiques.

TCMV (1930). Ortamektep Müfredat Programı. [Les programmes du collège]. Istanbul : Devlet Matbaası.

TCMV (1934). Lise Müfredat Programı. [Les programmes du lycée]. Istanbul : Devlet Matbaası.

YÜCEL, Hasan-Âli (1994 [1938]). Türkiye'de orta öğretim. [L'enseignement secondaire en Turquie]. Ankara : T.C Kültür Bakanlı̆̆ı. 


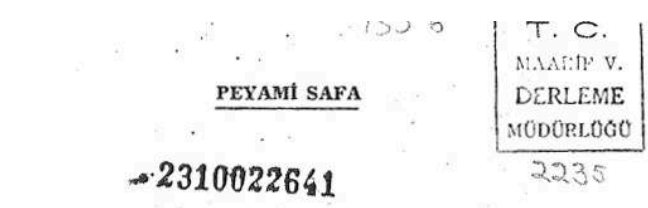

\title{
FRANSIZ GRAMERI
}

\author{
Okuma - Lûgat - Tercủme-Gramer \\ - Tatbikat ve Konusma \\ Tam gramerile birlikte fransizeayı kendi kendine \\ ögrenmek için en kolay metod \\ $194)$ AO 2235 \\ ALI TOYGAR \\ CUMHURIYET KITABEV \\ Istanbul Ankara caddesi No. 101 \\ D. 1.022641
}

\section{NOTES}

1. L'ouvrage s'intitule Grammaire du français auquel, dès l'édition suivante, s'ajoutera avec explications en turc.

2. Abdullah Cevdet (1869-1932) et son équipe sont des fervents défenseurs de la culture occidentale et déclarent ne pas en connaître d'autres.

3. École privée ouverte dans le yalı (pavillon) de Serasker Rıza Pasa, sur le Bosphore, à Vaniköy.

4. Sans exclure ses courtes traductions de Maupassant, de Rousseau et de La Rochefoucauld publiées dans les revues Servet-i Fünun, Fağfur,etc., nous pouvons citer à titre d'exemple: Üç Kardeş de Antoine Louis Camille Lemonnier, sous le pseudonyme de Server Bedi, Istanbul: Kitabhane-i Sudi, 1934 [1918] ; Engerek Düğümü de François Mauriac, Istanbul : Vakit, Gazete, Matbaa, Kütüphane, 1934 ; Açlık de Knut Hamsun, Istanbul, Resimli Ay Matbaası, 1934 ; Graziella de Lamartine, Istanbul : Semih Lütfi Kitabevi, 1939.

5. Les catégories grammaticales ou la métalangue avaient complètement changé entre-temps du fait des réformes linguistiques (purification des termes arabo-persans) et l'auteur a été obligé d'ajouter un lexique à la fin de son ouvrage pour harmoniser la terminologie avec celle utilisée dans les grammaires du turc.

6. Le nom, le genre, le nombre, l'article, l'adjectif, adjectifs déterminatifs, adjectifs numéraux ordinaux, adjectifs possessifs, etc. 


\section{RÉSUMÉS}

Le manuel de français préconisé par les programmes scolaires en 1940 en Turquie découle des méthodologies actives pour lesquelles l'objectif principal est l'acquisition d'un savoir lexical et grammatical sans avoir recours à la LM. Or, le sentiment général qui règne dans les milieux scolaires est que le français est une langue difficile et qu'on n'arrive pas à l'apprendre à l'école.

Fransiz grameri de Safa est une grammaire pédagogique qui se concentre également sur l'objet, mais propose une stratégie d'enseignement différente, à savoir le recours à la langue maternelle. Son livre qui éclaire chaque règle contraste avec les principes didactiques en vigueur, mais il assure une meilleure connaissance d'une L2 dont les concepts sont assez éloignés de la LM de l'apprenant.

The textbooks of French recommended by the official syllabuses for junior and senior highschool education in Turkey in 1940 follow the actual methodologies for which the major goal is the acquisition of a lexical and grammatical proficiency without having recourse to the mother tongue. Nonetheless, the general feeling that prevails over educational environments is that French is [an extremely] difficult language, and students fail to learn it.

Fransiz Grameri by the renowned Turkish novelist Peyami Safa is a grammar textbook which focuses likewise on the subject matter, but it suggests a different strategy of instruction. Namely, in this method learner is supposed to have recourse to the mother tongue. Safa's book that clarifies each [grammatical] rule, contrasts with current educational principles. However, it ensures a perfect comprehension of second language whose concepts are quite distant from those of the learner's mother tongue.

\section{INDEX}

Mots-clés : enseignement du français, grammaire, turc, manuel de français, Peyami Safa

Keywords : French language instruction, grammar, Turkish, textbook of French, Peyami Safa

\section{AUTEUR}

\section{HANIFE GÜVEN}

Université de Dokuz Eylül, Izmir, Turquie

hanife.guven@deu.edu.tr 